\title{
APPROXIMATE SOLUTION FOR EULER EQUATIONS OF STRATIFIED WATER VIA NUMERICAL SOLUTION OF COUPLED KdV SYSTEM
}

\author{
A. A. HALIM, S. P. KSHEVETSKII, and S. B. LEBLE
}

Received 23 December 2002

\begin{abstract}
We consider Euler equations with stratified background state that is valid for internal water waves. The solution of the initial-boundary problem for Boussinesq approximation in the waveguide mode is presented in terms of the stream function. The orthogonal eigenfunctions describe a vertical shape of the internal wave modes and satisfy a Sturm-Liouville problem. The horizontal profile is defined by a coupled KdV system which is numerically solved via a finite-difference scheme for which we prove the convergence and stability. Together with the solution of the Sturm-Liouville problem, the stream functions give the internal waves profile.

2000 Mathematics Subject Classification: 76B15, 76B70, 76B55, 37L65, 65M06, $65 \mathrm{M} 12$.
\end{abstract}

1. Introduction. The basic system of Euler equations for internal water waves in two dimensions $(x z)$, with a stable stratified ambient state and the buoyancy frequency $N(z)$, is

$$
\begin{gathered}
u_{x}+w_{z}=0, \\
\rho_{o} u_{t}=-\rho_{o}(\vec{v}, \vec{\nabla}) u-p_{x}, \\
\rho_{o} w_{t}=-\rho_{o}(\vec{v}, \vec{\nabla}) w-p_{z}-\rho^{\prime} g, \\
T_{t}^{\prime}+w \bar{T}_{z}=-(\vec{v}, \vec{\nabla}) T^{\prime},
\end{gathered}
$$

where $u, v$ are velocity components, $\rho_{o}$ is the density, $p$ is the pressure, $\rho^{\prime} g$ is the body force due to stratification, $\bar{T}_{z}$ is the vertical background temperature gradient, and $T^{\prime}$ is the temperature variable [3]. Combining the equations in (1.1) and using the state relation for liquid $\rho^{\prime}=-\rho_{o} \alpha T^{\prime}, \alpha=-\rho_{z} /\left(\rho \bar{T}_{z}\right)$ is the coefficient of thermal expansion, and $\bar{T}_{z}=N^{2} /(\alpha g)$, we obtain

$$
\begin{aligned}
\Delta w_{t t}+ & N^{2} w_{x x}-N^{2}\left[(\vec{v}, \vec{\nabla}) \int_{0}^{t} w d t\right]_{x x} \\
& +\left[(\vec{v}, \vec{\nabla}) \int w_{z} d x\right]_{t x z}+[(\vec{v}, \vec{\nabla}) w]_{t x x}=0 .
\end{aligned}
$$


Rescaling the dimensionless variables (primed) using $x_{i}=\lambda_{i} x_{i}^{\prime}, t=2 \pi t^{\prime} / \bar{N} \beta$, $u=\lambda_{z} \bar{N} u^{\prime} / 2 \pi, w=\beta \lambda_{z} \bar{N} w^{\prime} / 2 \pi, \alpha T^{\prime}=T, N=\bar{N} N^{\prime} / 2 \pi, \bar{N}$ is the average buoyancy frequency and $\beta$ is a scale parameter. Substitute in (1.2) and omit primes for simplicity to obtain

$$
\begin{array}{r}
\lambda_{z}\left(\frac{\bar{N} \beta}{2 \pi}\right)^{3}\left(\frac{w_{x x}}{\lambda_{x}^{2}}+\frac{w_{z z}}{\lambda_{z}^{2}}\right)_{t t}+\left(\frac{\bar{N}}{2 \pi}\right)^{3}(\beta N)^{2}\left(\frac{w_{x x}}{\lambda_{x}}\right) \\
-\left(\frac{\bar{N}}{2 \pi}\right)^{3}\left(\frac{(\beta N)^{2}}{\lambda_{x}}\right)\left[u \int_{0}^{t} w_{x} d t+w \int_{0}^{t} w_{z} d t\right]_{x x} \\
+\left(\frac{\bar{N} \beta}{2 \pi}\right)^{3}\left\{\frac{\lambda_{z}}{\lambda_{x}^{2}}\left[u w_{x}+w w_{z}\right]_{x x t}\right. \\
\left.+\frac{1}{\lambda_{z}}\left[u w_{z}+w\left(\int_{0}^{t} w_{z} d x\right)_{z}\right]_{x z t}\right\}=0 .
\end{array}
$$

Introduce the stream function $\psi, w=-\sigma \psi_{x}$ and $u=\sigma \psi_{z}$ ( $\sigma$ is a scale parameter). Integrate, with respect to $x$,

$$
\begin{aligned}
\psi_{z z t t}+N^{2} \psi_{x x}= & -\beta^{2} \psi_{x x t t}-\sigma\left[\psi_{z} \psi_{x z}-\psi_{x} \psi_{z z}\right]_{z t} \\
& +\sigma N^{2}\left[\psi_{z} \int_{0}^{t} \psi_{x x} d t-\psi_{x} \int_{0}^{t} \psi_{x z} d t\right]_{x} .
\end{aligned}
$$

Substitute in (1.4) by the stream function of the form

$$
\psi(z, x, t)=\sum_{m} Z^{m}(z) \theta^{m}(x, t)
$$

multiply by $Z^{n}$, integrate with respect to $z$, and use the separation of variables that give

$$
Z_{z z}^{n}=-\frac{N^{2}}{c_{n}^{2}} Z^{n}
$$

So (1.4) becomes

$$
\begin{aligned}
\theta_{t t}^{n} & -c_{n}^{2} \theta_{x x}^{n} \\
& =\frac{c_{n}^{2} \beta^{2}}{N^{2}} \theta_{x x t t}^{n}+\sigma c_{n}^{2} \sum_{m, k}\left\{a_{m, k}^{n}\left(\theta^{m} \theta_{x}^{k}\right)_{t}+\left[b_{m, k}^{n} \theta^{m} \theta_{t}^{k}+e_{m, k}^{n} \theta_{x}^{m} \int_{0}^{t} \theta_{x}^{k} d t\right]_{x}\right\},
\end{aligned}
$$


where

$$
\begin{gathered}
a_{m, k}^{n}=N^{2} \int_{-h}^{h}\left(\frac{-1}{c_{k}^{2}}+\frac{1}{c_{m}^{2}}\right) z_{z}^{m} z^{k} z^{n} d z, \quad b_{m, k}^{n}=\frac{-N^{2}}{c_{k}^{2}} \int_{-h}^{h} z_{z}^{m} z^{k} z^{n} d z, \\
e_{m, k}^{n}=N^{2} \int_{-h}^{h} z^{m} z_{z}^{k} z^{n} d z .
\end{gathered}
$$

System (1.7) describes the two oppositely directed propagated modes. The equations of the separated propagated modes are obtained by substituting $\theta_{t}^{n}=u^{n}, c_{n} \theta_{x}^{n}=v^{n}$, so (1.7) becomes

$$
\begin{aligned}
u_{t}^{n}-c_{n} v_{x}^{n}=\frac{c_{n}^{3} \beta^{2}}{N^{2}} v_{x x x}^{n}+\sigma c_{n}^{2} \sum_{m, k}\{ & a_{m, k}^{n}\left(\int_{0}^{t} u^{m} d t \cdot \frac{v^{k}}{c_{k}}\right)_{t} \\
+ & {\left.\left[b_{m, k}^{n} \int_{0}^{t} u^{m} d t \cdot u^{k}+e_{m, k}^{n} \frac{v^{m}}{c_{m}} \int_{0}^{t} \frac{v^{k}}{c_{k}} d t\right]_{x}\right\}, }
\end{aligned}
$$

$v_{t}^{n}-c_{n} u_{x}^{n}=0$.

Using projection operators,

$$
\begin{aligned}
& P_{+}=\frac{1}{2}\left(\begin{array}{cc}
1 & 1+\frac{c^{2} \beta^{2}}{2 N^{2}} \partial_{x}^{2} \\
1-\frac{c^{2} \beta^{2}}{2 N^{2}} \partial_{x}^{2} & 1
\end{array}\right), \\
& P_{-}=\frac{1}{2}\left(\begin{array}{cc}
1 & -1-\frac{c^{2} \beta^{2}}{2 N^{2}} \partial_{x}^{2} \\
-1+\frac{c^{2} \beta^{2}}{2 N^{2}} \partial_{x}^{2} & 1
\end{array}\right) .
\end{aligned}
$$

So

$$
P_{+}\left(\begin{array}{c}
u^{n} \\
v^{n}
\end{array}\right)=\left(\begin{array}{c}
\varphi^{n+} \\
k \varphi^{n+}
\end{array}\right), \quad P_{-}\left(\begin{array}{c}
u^{n} \\
v^{n}
\end{array}\right)=\left(\begin{array}{c}
\varphi^{n-} \\
-k \varphi^{n-}
\end{array}\right)
$$

or

$$
u^{n}=\varphi^{n+}+\varphi^{n-}, \quad v^{n}=\left(\varphi^{n+}-\varphi^{n-}\right)-\frac{c^{2} \beta^{2}}{2 N^{2}} \partial_{x}^{2}\left(\varphi^{n+}-\varphi^{n-}\right) .
$$

Operating $P_{+}, P_{-}$on (1.9) and using (1.12), we obtain the equations for the separated modes $\varphi^{n+}, \varphi^{n-}$ as

$$
\begin{gathered}
\varphi_{t}^{n+}-c_{n} \varphi_{x}^{n+}-\frac{c^{3} \beta^{2}}{2 N^{2}} \varphi_{x x x}^{n+}-\frac{\sigma c_{n}^{2}}{2} \sum_{m, k} a_{m, k}^{n}\left(\int_{0}^{t}\left(\varphi^{+}+\varphi^{-}\right)^{m} d t \cdot \frac{\left(\varphi^{+}-\varphi^{-}\right)^{k}}{c_{k}}\right)_{t} \\
+\left[b_{m, k}^{n} \int_{0}^{t}\left(\varphi^{+}+\varphi^{-}\right)^{m} d t \cdot\left(\varphi^{+}+\varphi^{-}\right)^{k}\right. \\
\left.+e_{m, k}^{n} \frac{\left(\varphi^{+}-\varphi^{-}\right)^{m}}{c_{m}} \int_{0}^{t} \frac{\left(\varphi^{+}-\varphi^{-}\right)^{k}}{c_{k}} d t\right]_{x}=0,
\end{gathered}
$$




$$
\begin{gathered}
\varphi_{t}^{n-}+c_{n} \varphi_{x}^{n-}+\frac{c^{3} \beta^{2}}{2 N^{2}} \varphi_{x x x}^{n-}-\frac{\sigma c_{n}^{2}}{2} \sum_{m, k} a_{m, k}^{n}\left(\int_{0}^{t}\left(\varphi^{+}+\varphi^{-}\right)^{m} d t \cdot \frac{\left(\varphi^{+}-\varphi^{-}\right)^{k}}{c_{k}}\right)_{t} \\
+\left[b_{m, k}^{n} \int_{0}^{t}\left(\varphi^{+}+\varphi^{-}\right)^{m} d t \cdot\left(\varphi^{+}+\varphi^{-}\right)^{k}\right. \\
\left.+e_{m, k}^{n} \frac{\left(\varphi^{+}-\varphi^{-}\right)^{m}}{c_{m}} \int_{0}^{t} \frac{\left(\varphi^{+}-\varphi^{-}\right)^{k}}{c_{k}} d t\right]_{x}=0
\end{gathered}
$$

Let $\varphi^{n+}=\eta_{t}^{n+}, \varphi^{n-}=\eta_{t}^{n-}$, so (1.13) becomes

$$
\begin{aligned}
\eta_{t}^{n+}-c_{n} \eta_{x}^{n+}= & \frac{c^{3} \beta^{2}}{2 N^{2}} \eta_{x x x}^{n+}+\frac{\sigma c_{n}^{2}}{2} \sum_{m, k} a_{m, k}^{n}\left(\eta^{+}+\eta^{-}\right)^{m}\left(\eta^{+}-\eta^{-}\right)_{x}^{k} \\
& +b_{m, k}^{n}\left(\eta^{+}+\eta^{-}\right)^{m} \cdot\left(\eta^{+}+\eta^{-}\right)_{x}^{k} \\
& +\frac{e_{m, k}^{n}}{c_{k} c_{m}}\left(\eta^{+}-\eta^{-}\right)_{x}^{m}\left(\eta^{+}-\eta^{-}\right)^{k}, \\
\eta_{t}^{n-}+c_{n} \eta_{x}^{n-}= & -\frac{c^{3} \beta^{2}}{2 N^{2}} \eta_{x x x}^{n-}+\frac{\sigma c_{n}^{2}}{2} \sum_{m, k} a_{m, k}^{n}\left(\eta^{+}+\eta^{-}\right)^{m}\left(\eta^{+}-\eta^{-}\right)_{x}^{k} \\
& +b_{m, k}^{n}\left(\eta^{+}+\eta^{-}\right)^{m} \cdot\left(\eta^{+}+\eta^{-}\right)_{x}^{k} \\
& +\frac{e_{m, k}^{n}}{c_{k} c_{m}}\left(\eta^{+}-\eta^{-}\right)_{x}^{m}\left(\eta^{+}-\eta^{-}\right)^{k} .
\end{aligned}
$$

In what follows we will consider both directed modes. In this work we evaluate only for short time to describe the phenomena, so we consider only one direction of the propagating modes. Hence the system that describes one direction of (1.7) has the form (return to $\theta$ variable for convenient)

$$
\begin{gathered}
\theta_{t}^{n}+c_{n} \theta_{x}^{n}+\sigma \sum_{m, k} g_{m, k}^{n} \theta^{m} \theta_{x}^{k}+\beta^{2} d_{n} \theta_{x x x}^{n}=0 \\
g_{m, k}^{n}=\frac{\sigma N^{2} c_{n}^{2}}{2} \int_{-h}^{h}\left[\left(\frac{-1}{c_{m}^{2}}+\frac{2}{c_{k}^{2}}\right) Z^{k} Z_{z}^{m}-\frac{1}{c_{m} c_{k}} Z^{m} Z_{z}^{k}\right] Z^{n} d z, \quad d_{n}=\frac{c_{n}^{3}}{2 N^{2}}
\end{gathered}
$$

Hence we can summarize as follows: in the commonly accepted approximations (incompressibility, Boussinesq approximation, and so forth) the solution of system (1.1) is constructed as the representation for the stream function (1.5) which is completely defined by solving (1.6) and (1.15). 
2. Physical model. In this model, we simulate the initial stage of McEwan experiment $[4,5]$ for a rectangular tank of dimension $50 \mathrm{~cm}$ in $x$ by $25 \mathrm{~cm}$ in $z$ directions filled by a linearly stratified water of constant buoyancy frequency $1.23 \mathrm{~s}^{-1}$. The internal water waves are described by system (1.1). The solution of this system is constructed as the representation for the stream function (1.5), where $Z^{n}(z)$ are solutions of the correspondent Sturm-Liouville problem (1.6), $Z_{z z}+\left(N^{2} / c_{n}^{2}\right) Z=0, Z(0)=Z(h)=0$, and describe a vertical shape of the wave modes. The linear propagation velocities $c_{n}$ play the role of eigenvalues. The coefficient functions $\theta^{n}(x, t)$ are solutions of the coupled KdV system (1.15) with coefficients from (1.16). We select a localized initial condition along $x$-axis described by a smooth enough function that models the paddle motion as in the experiments of McEwan [5]. The function is also chosen antisymmetric along $x$-axis in relation to the paddle axis centered in the middle of the tank. The time intervals of simulations are taken such that the initial disturbance decays essentially but does not reach the boundaries.

3. Computation. When the coefficients in the Sturm-Liouville equation (1.6) are constant, it has very simple general solution

$$
Z^{n}=B_{n} \sin \left(\frac{n \pi z}{h}\right), \quad n=1,2,3, \ldots, L
$$

which tends to zero at boundaries. The eigenvalues

$$
c_{n}=\frac{N h}{n \pi}, \quad n=1,2,3, \ldots, L,
$$

have the sense of linear internal gravity waves velocities. Normalization is determined by $\int_{0}^{h}\left(Z^{n}\right)^{2} N^{2} d z=1$ and gives $B_{n}=\left(2 / N^{2} h\right)^{1 / 2}$. Hence

$$
Z^{n}(z)=\left(\frac{2}{N^{2} h}\right)^{1 / 2} \sin \left(\frac{n \pi z}{h}\right)
$$

To solve the coupled KdV system (1.15) an initial condition is required. We can select the initial perturbation for the stream function (1.5) which has the general form

$$
\psi(z, x, 0)=\sum_{n=1}^{L} Z^{n}(z) \theta^{n}(x, 0)=\varphi(x, z)=\varphi_{1}(x) \varphi_{2}(z) .
$$

Taking the exponentially localized functions $\varphi_{1}(x), \varphi_{2}(z)$ : 


$$
\begin{gathered}
\varphi_{1}(x)=\frac{a}{\cosh (x / l)}, \\
\varphi_{2}(z)=\left(\frac{2}{N^{2} h}\right)^{1 / 2} \operatorname{sech}\left(b\left(z-z_{0}\right)\right) \tanh \left(b\left(z-z_{0}\right)\right),
\end{gathered}
$$

as the initial condition we model the impact of a wave-productor.

The choice of the form and the constants $a, b, l$ qualitatively reflects the paddle movement (we restrict the movement by some isolated pulse), and the numerical value for the amplitude is estimated also from the description of the experiment of McEwan [5]. Then the scalar product gives

$$
\begin{aligned}
\left(Z^{j}, \psi\right)= & \sum_{n=1}^{L}\left(Z^{j}, Z^{n}\right) \theta^{n}(x, 0)=\left(Z^{j}, \varphi_{2}(z)\right) \varphi_{1}(x) \\
& \left(Z^{j}, \varphi_{2}(z)\right)=\int_{0}^{h} N^{2} Z^{j} \varphi_{2}(z) d z
\end{aligned}
$$

and using the orthogonality,

$$
\left(Z^{j}, Z^{n}\right)=\int_{0}^{h} N^{2} Z^{j} Z^{n} d z=\{1,(j=n)\},\{0,(j \neq n)\} .
$$

Hence, (3.6) gives $\theta^{n}(x, 0)$, the initial condition of system (1.15) which is solved by the numerical method introduced below.

4. Numerical method. For the coupled KdV system (1.15) we introduce a numerical (finite-difference) method of solution, a two-step three-time-level scheme similar to the Lax-Wendroff one $[1,7]$. The usual Lax-Wendroff scheme is modified such that the order of the first derivative becomes of order $O\left(\triangle x^{4}\right)$. The approximation of the nonlinear terms is changed such that the integral of $\theta^{2}$ is a conserved one. The scheme has the form

$$
\begin{aligned}
& \frac{\left(\left(\theta^{n}\right)_{i}^{j+1 / 2}-\left(\theta^{n}\right)_{i}^{j}\right)}{\tau / 2}+\frac{c_{n}\left(\left(\theta^{n}\right)_{i+1}^{j}-\left(\theta^{n}\right)_{i-1}^{j}\right)}{2 h} \\
& \quad+\sum_{k, m} g_{m k}^{n}\left(\theta^{m}\right)_{i}^{j} \frac{\left(\left(\theta^{k}\right)_{i+1}^{j}-\left(\theta^{k}\right)_{i-1}^{j}\right)}{2 h} \\
& \quad+\left(d_{n}-\frac{c_{n} h^{2}}{6}\right) \frac{\left(\left(\theta^{n}\right)_{i+2}^{j}-2\left(\theta^{n}\right)_{i+1}^{j}+2\left(\theta^{n}\right)_{i-1}^{j}-\left(\theta^{n}\right)_{i-2}^{j}\right)}{2 h^{3}} \\
& =0,
\end{aligned}
$$

where $n, m$, and $k$ are the modes numbers and $i$ and $j$ are discrete space and time variables, respectively. The time step is denoted by $\tau$ while the spatial step size is denoted by $h$. Equation (4.1) is accompanied by a discrete equation 
for the intermediate layer as

$$
\begin{aligned}
& \frac{\left(\left(\theta^{n}\right)_{i}^{j+1}-\left(\theta^{n}\right)_{i}^{j}\right)}{\tau}+\frac{c_{n}\left(\left(\theta^{n}\right)_{i+1}^{j+1 / 2}-\left(\theta^{n}\right)_{i-1}^{j+1 / 2}\right)}{2 h} \\
& \quad+\sum_{k, m} g_{m k}^{n}\left(\theta^{m}\right)_{i}^{j+1 / 2} \frac{\left(\left(\theta^{k}\right)_{i+1}^{j+1 / 2}-\left(\theta^{k}\right)_{i-1}^{j+1 / 2}\right)}{2 h} \\
& \quad+\frac{e_{n}\left(\left(\theta^{n}\right)_{i+2}^{j+1 / 2}-2\left(\theta^{n}\right)_{i+1}^{j+1 / 2}+2\left(\theta^{n}\right)_{i-1}^{j+1 / 2}-\left(\theta^{n}\right)_{i-2}^{j+1 / 2}\right)}{2 h^{3}}=0 .
\end{aligned}
$$

To support the results, we prove stability and convergence of the scheme in Appendices A and B [2]. Beside these proofs, we would like to mention that the order of errors of the difference formulas is improved at the same time they preserve conservation laws of the KdV-type equations [6].

5. Results. Figure 5.1 is a contour plot presenting the initial perturbation $\psi(z, x, 0)$ for the upper half of the tank due to antisymmetry.

Figure 5.2 is one-dimensional $(x)$ plots for the second tenth modes.

Figure 5.3 is a three-dimensional plot for the wave profile (1.5) at $t=0.02$ second for the same half of the tank.

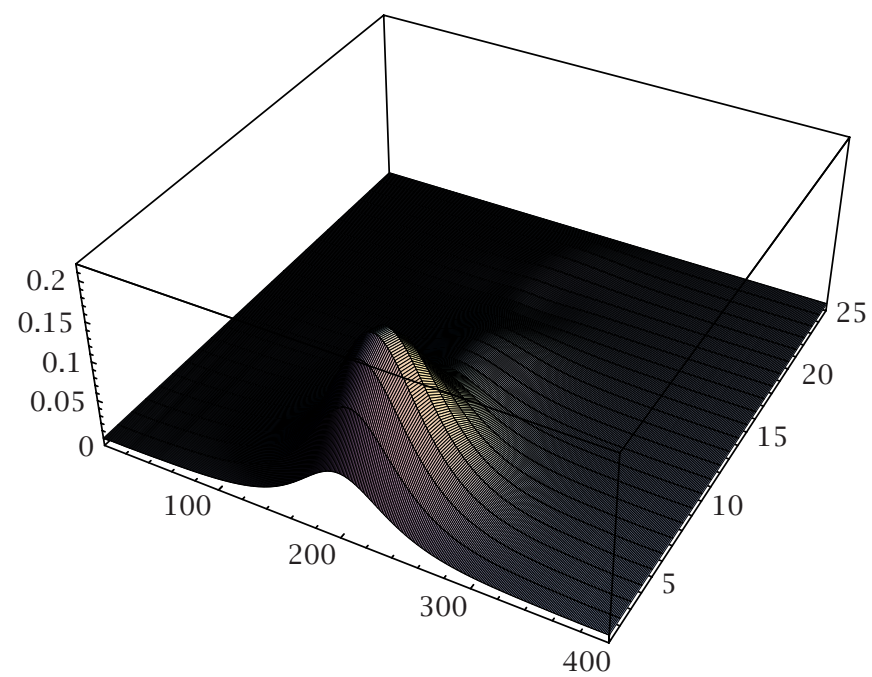

FIGURE 5.1. Three-dimensional plot for the initial perturbation $\psi(z, x, 0)$ for the upper half of the tank. The horizontal numbers $(25,400)$ indicate the number of mesh points used in plotting in $x$ and $z$ directions while the dimensions are $12.5 \mathrm{~cm}$ in $z$ and $50 \mathrm{~cm}$ in $x$, respectively. 


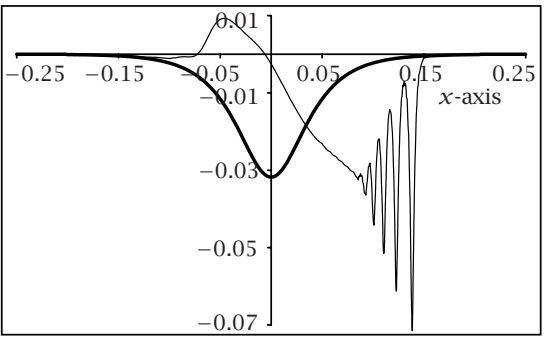

Second mode, series $1(t=0)$, series $2(t=0.02 \mathrm{~s})$

- Series 1

- Series 2

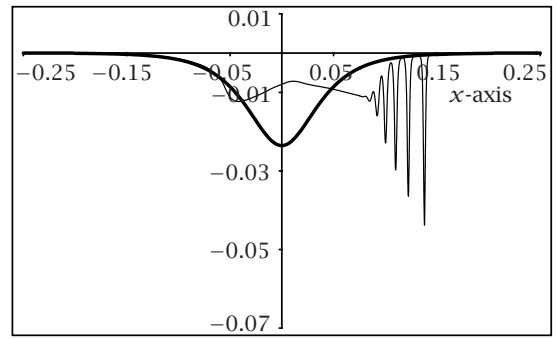

Sixth mode, series $1(t=0)$, series $2(t=0.02 \mathrm{~s})$

- Series 1

- Series 2

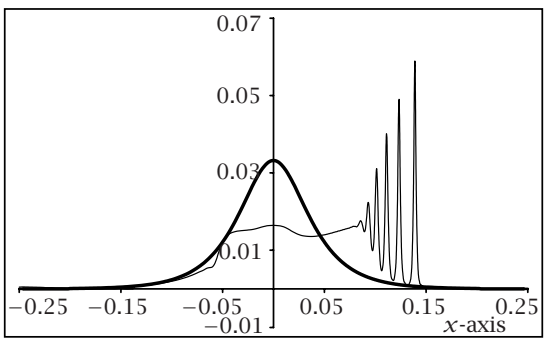

Fourth mode, series $1(t=0)$, series $2(t=0.02 \mathrm{~s})$

\section{- Series 1}

- Series 2

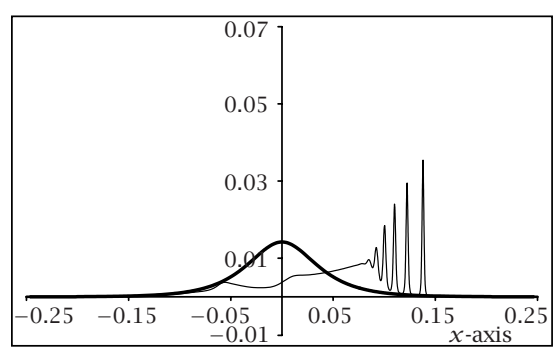

Eighth mode, series $1(t=0)$, series $2(t=0.02 \mathrm{~s})$

- Series 1

— Series 2

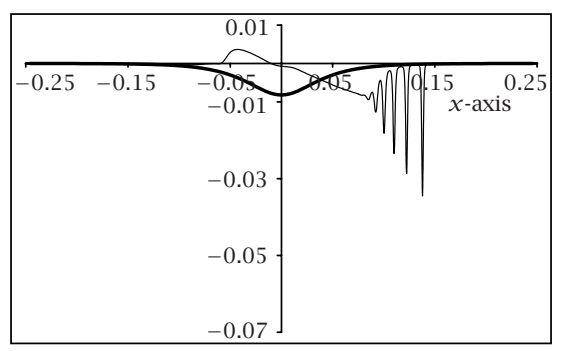

Tenth mode, series $1(t=0)$, series $2(t=0.02 \mathrm{~s})$

- Series 1

- Series 2

FIGURE 5.2. One-dimensional ( $x$ ) plots of the wave profile at $t=0$ and $t=0.02$ second. 


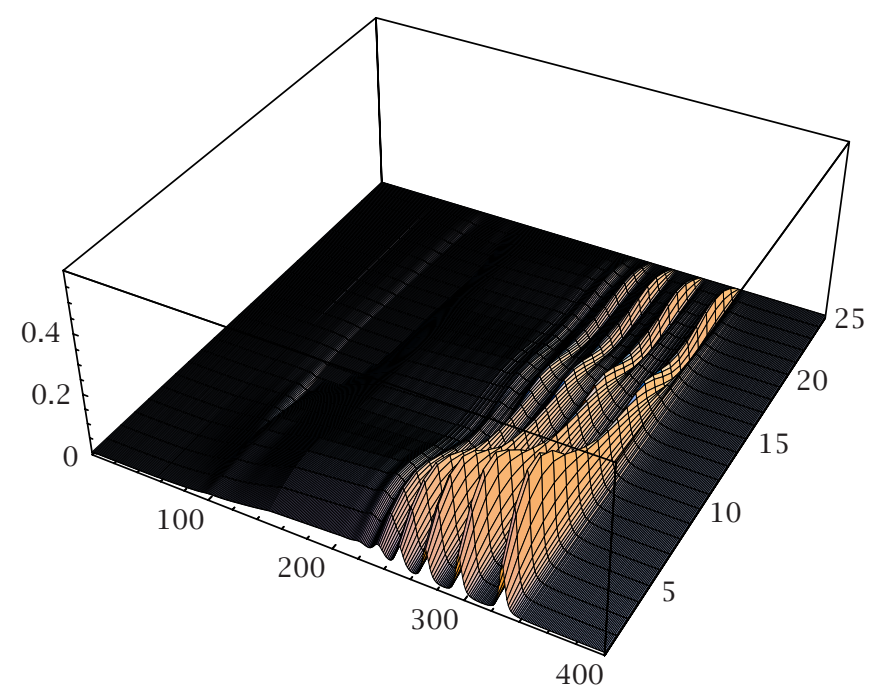

FIGURE 5.3. Three-dimensional $(x, y, z)$ plot of the wave profile $\psi(z, x, t)$ at $t=0.02$ second for the upper half of the tank. The horizontal numbers $(25,400)$ indicate the number of mesh points used in plotting in $x$ and $z$ directions while the dimensions are $12.5 \mathrm{~cm}$ in $z$ and $50 \mathrm{~cm}$ in $x$, respectively.

All the plots for the modes and for the sum (stream function) show the behavior that is typical for the multisolitonic perturbation. It looks like a decay of the initial condition to solitons in the single KdV equation theory. The process of the wave propagation is accompanied by interaction that implies the energy transfer between modes. This phenomenon may be considered as a possible reason for the vertical fine-structure generation [3]. The combination of the fine structures may explain McEwan experiments [4, 5].

6. Summary. In the commonly accepted approximations (incompressibility, Boussinesq approximation, and so forth), the solution of the system of Euler equations with stratified background state is constructed as the representation for the stream function. The horizontal profile is defined by a coupled KdV system which is numerically solved via a finite-difference scheme for which stability and convergence are proved. Together with the solution of the Sturm-Liouville problem that describes the vertical profile, the stream function gives the complete internal waves profile.

\section{Appendices}

A. Stability proof of the scheme. We prove stability with respect to small perturbations (because we consider nonlinear equations) of initial conditions. Strictly speaking, it is the boundness of the discrete solution in terms of small 
perturbation of the initial data. Consider the differential

$$
\begin{gathered}
T_{i, r}^{n, j+1}=\left\{\frac{\partial \theta_{i}^{n, j+1}}{\partial \theta_{r}^{n, j}}\right\}, \\
d \theta_{r}^{n, j}=\left(\begin{array}{lllll}
\theta_{i-2}^{n, j} & \theta_{i-1}^{n, j} & \theta_{i}^{n, j} & \theta_{i+1}^{n, j} & \theta_{i+2}^{n, j}
\end{array}\right)^{t}
\end{gathered}
$$

and define the norm

$$
\left\|d \theta^{j}\right\|=\left(\sum_{r} \sum_{n}\left(d \theta_{r}^{n, j}\right)^{2} h\right)^{1 / 2} .
$$

We can write

$$
d \theta_{i}^{n, j+1}=T_{i, r}^{n, j+1} d \theta_{r}^{n, j}=T_{i, r}^{n, j+1} T_{i, r}^{n, j} d \theta_{r}^{n, j-1}=\Pi_{r}\left(T_{i, r}^{n}\right)^{r} d \theta_{r}^{n, o},
$$

where $d \theta_{i}^{n, j+1}$ is the perturbation of the discrete solution and $d \theta_{r}^{n, o}$ is a small perturbation of the initial data. Stability required the boundness of $\Pi_{r}\left(T_{i, r}^{n}\right)^{r}$, that is, $\left\|T^{r}\right\|$ is bounded. We calculate $T$ from the difference scheme as follows:

$$
\begin{aligned}
T_{i, r}^{n, j+1}= & \delta_{i, r}-\frac{c_{n} T}{2 h}\left[\delta_{i+1, r}-\delta_{i-1, r}\right] \\
& -\tau \sum_{m, k}\left\{\frac{g_{m, k}^{n}}{2 h}\left[\theta_{i}^{m, j}\left(\delta_{i+1, r}-\delta_{i-1, r}\right)+\delta_{i, r}\left(\theta_{i+1}^{k, j}-\theta_{i-1}^{k, j}\right)\right]\right\} \\
& -\frac{\tau e_{n}}{2 h^{3}}\left[\delta_{i+2, r}-2 \delta_{i+1, r}+2 \delta_{i-1, r}-2 \delta_{i-2, r}\right] .
\end{aligned}
$$

Rewriting the matrix $T$ (A.4) in terms of identity $(E)$, symmetric $(S)$, and antisymmetric $(A)$, matrices yields $T=E+S+A$,

$$
\begin{aligned}
\left\{S^{n, j+1}\right\}_{i, r}= & -\frac{\tau}{4 h} \sum_{m, k} g_{m, k}^{n}\left[\left(\theta_{i}^{m, j}-\theta_{i+1}^{m, j}\right) \delta_{i+1, r}-\left(\theta_{i}^{m, j}-\theta_{i-1}^{m, j}\right) \delta_{i-1, r}\right. \\
& \left.+2 \delta_{i, r}\left(\theta_{i+1}^{k, j}-\theta_{i-1}^{k, j}\right)\right], \\
\left\{A^{n, j+1}\right\}_{i, r}= & -\frac{c_{n} T}{2 h}\left[\delta_{i+1, r}-\delta_{i-1, r}\right] \\
& -\frac{\tau}{4 h} \sum_{m, k} g_{m, k}^{n}\left[\left(\theta_{i}^{m, j}+\theta_{i+1}^{m, j}\right) \delta_{i+1, r}-\left(\theta_{i}^{m, j}+\theta_{i-1}^{m, j}\right) \delta_{i-1, r}\right] \\
& -\frac{e_{n} \tau}{2 h^{3}}\left[\delta_{i+2, r}-2 \delta_{i+1, r}+2 \delta_{i-1, r}-\delta_{i-2, r}\right], \\
\left\|S^{j+1}\right\| \leq & \tau \max _{n, m, k}\left|g_{m, k}^{n}\right| \max _{i, m, k}\left(\left|\theta_{x, i}^{m, j}\right|,\left|\theta_{x, i}^{k, j}\right|\right),
\end{aligned}
$$




$$
\begin{aligned}
& \theta_{x, i}^{m, j}=\frac{\left(\theta_{i+1}^{m, j}-\theta_{i}^{m, j}\right)}{h}, \quad \theta_{x, i}^{k, j}=\frac{\left(\theta_{i+1}^{k, j}-\theta_{i-1}^{k, j}\right)}{2 h}, \\
& \left\|A^{j+1}\right\| \leq \frac{\tau \max _{n, m, k}\left|g_{m, k}^{n}\right|}{h} \max _{m, i}\left|\theta_{i}^{m, j}\right|+\frac{\tau \max _{n}\left|c_{n}\right|}{h}+\frac{3 \tau \max _{n}\left|e_{n}\right|}{h^{3}} \\
& \left\|T^{j+1}\right\|^{2}=\left\|\left(T^{j+1}\right)^{*} T^{j+1}\right\|=\left\|\left(E-A^{j+1}+S^{j+1}\right)\left(E+A^{j+1}+S^{j+1}\right)\right\| \\
& \leq 1+2\left\|S^{j+1}\right\|+\left(\left\|A^{j+1}\right\|+\left\|S^{j+1}\right\|\right)^{2} \\
& \leq 1+2 \tau \max _{n, m, k}\left|g_{m, k}^{n}\right| \max _{m, i}\left|\theta_{x, i}^{m, j}\right| \\
& +\tau^{2}\left(\max _{n, m, k}\left|g_{m, k}^{n}\right| \max _{m, i}\left|\theta_{x, i}^{m, j}\right|+\frac{\max _{n, m, k}\left|g_{m, k}^{n}\right|}{h} \max _{m, i}\left|\theta_{i}^{m, j}\right|\right. \\
& \left.+\frac{\max _{n}\left|c_{n}\right|}{h}+\frac{3 \max _{n}\left|e_{n}\right|}{h^{3}}\right)^{2} \\
& \leq e^{a \tau} \\
& a=1+2 \tau \max _{n, m, k}\left|g_{m, k}^{n}\right| \max _{m, i}\left|\theta_{x, i}^{m, j}\right| \\
& +\tau^{2}\left(\max _{n, m, k}\left|g_{m, k}^{n}\right| \max _{m, i}\left|\theta_{x, i}^{m, j}\right|+\frac{\max _{n, m, k}\left|g_{m, k}^{n}\right|}{h} \max _{m, i}\left|\theta_{i}^{m, j}\right|\right. \\
& \left.+\frac{\max _{n}\left|c_{n}\right|}{h}+\frac{3 \max _{n}\left|e_{n}\right|}{h^{3}}\right)^{2}
\end{aligned}
$$

which is a necessary condition of stability. The scheme is stable if $a \leq$ constant in spite of $\tau, h \rightarrow 0$. This is a conditional stability of the scheme. It means that it is required for stability that $\tau \rightarrow 0$ more faster than $h \rightarrow 0$ or

$$
\tau \leq(\text { constant }) \cdot h^{6} .
$$

B. Convergence proof of the scheme. We would prove that the solution of the difference equations (4.1) and (4.2) converges to the solution of (1.15) if the exact solution is a continuously differentiable one. We place here for brevity a proof that uses only one-step time difference equation and the whole proof may be developed by similar ideas. Therefore, we now consider the difference equation

$$
\begin{gathered}
\frac{\left(\theta^{n}\right)_{i}^{j+1}-\left(\theta^{n}\right)_{i}^{j}}{\tau}+c_{n} \frac{\left(\theta^{n}\right)_{i+1}^{j}-\left(\theta^{n}\right)_{i-1}^{j}}{2 h}+\sum_{m, k} g_{m, k}^{n}\left(\theta^{m}\right)_{i}^{j} \frac{\left(\theta^{k}\right)_{i+1}^{j}-\left(\theta^{k}\right)_{i-1}^{j}}{2 h} \\
+e_{n} \frac{\left(\theta^{n}\right)_{i+2}^{j}-2\left(\theta^{n}\right)_{i+1}^{j}+2\left(\theta^{n}\right)_{i-1}^{j}-\left(\theta^{n}\right)_{i-2}^{j}}{2 h^{3}}=0 .
\end{gathered}
$$


We know that the KdV-type equation has the conservation law $\int_{-\infty}^{\infty} u^{2}(x, t) d x=$ constant. It may also be shown that if a smooth enough solution $u^{n}(x, t)$ of the set of equations (1.15) exists, then it satisfies the inequality $\sum_{n=1}^{L} \int_{-\infty}^{\infty}\left(u^{n}(x\right.$, $t))^{2} d x<B$, for a finite $t$, where $B$ is a constant dependent on initial conditions only, $L$-number of modes taken into account by system (1.15). Therefore, it is natural to use $L_{2}$-norm in the proof, and

$$
\left\|\Theta^{j}\right\|=\left(\sum_{i} \sum_{n}\left[\left(\theta^{n}\right)^{2} h\right]_{i}^{j}\right)^{1 / 2} .
$$

Here, $h$ is a grid step, $i$ is a discrete space variable, and $j$ is the discrete time.

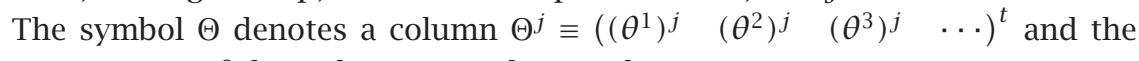
components of this column are columns also:

$$
\left(\theta^{n}\right)^{j} \equiv\left(\cdots \quad\left(\theta^{n}\right)_{i-1}^{j} \quad\left(\theta^{n}\right)_{i}^{j} \quad\left(\theta^{n}\right)_{i+1}^{j} \quad \cdots\right)^{t}
$$

where $\left(\theta^{n}\right)_{i}^{j}$ is a solution of the finite-difference equation (B.1).

Let the vector $\left(u^{n}\right)_{i}^{j}=u^{n}\left(x_{i}, t^{j}\right)$ be an exact solution of (B.1) in the points of grid. Then the error $\left(v^{n}\right)_{i}^{j}$ is given by

$$
\left(v^{n}\right)_{i}^{j}=\left(\theta^{n}\right)_{i}^{j}-\left(u^{n}\right)_{i}^{j} .
$$

The difference solution $\left(\theta^{n}\right)_{i}^{j}$ converges to the exact solution $\left(u^{n}\right)_{i}^{j}$ if $\left\|V^{j}\right\| \rightarrow 0$ as $\tau, h \rightarrow 0$, where $V^{j} \equiv \Theta^{j}-U^{j}$. Substitute (B.4) into (B.1) and obtain

$$
\begin{aligned}
& \frac{\left(v^{n}\right)_{i}^{j+1}-\left(v^{n}\right)_{i}^{j}}{\tau}+c_{n} \frac{\left(v^{n}\right)_{i+1}^{j}-\left(v^{n}\right)_{i-1}^{j}}{2 h} \\
& +\sum_{m, k}\left(g_{m, k}^{n}\left(u^{m}\right)_{i}^{j} \frac{\left(v^{k}\right)_{i+1}^{j}-\left(v^{k}\right)_{i-1}^{j}}{2 h}+g_{m, k}^{n}\left(v^{m}\right)_{i}^{j} \frac{\left(u^{k}\right)_{i+1}^{j}-\left(u^{k}\right)_{i-1}^{j}}{2 h}\right. \\
& \left.\quad+g_{m, k}^{n}\left(v^{m}\right)_{i}^{j} \frac{\left(v^{k}\right)_{i+1}^{j}-\left(v^{k}\right)_{i-1}^{j}}{2 h}\right) \\
& +e_{n} \frac{\left(v^{n}\right)_{i+2}^{j}-2\left(v^{n}\right)_{i+1}^{j}+2\left(v^{n}\right)_{i-1}^{j}-\left(v^{n}\right)_{i-2}^{j}}{2 h^{3}} \\
& =-\left(\frac{\left(u^{n}\right)_{i}^{j+1}-\left(u^{n}\right)_{i}^{j}}{\tau}+c_{n} \frac{\left(u^{n}\right)_{i+1}^{j}-\left(u^{n}\right)_{i-1}^{j}}{2 h}\right. \\
& \quad+\sum_{m, k} g_{m, k}^{n}\left(u^{m}\right)_{i}^{j} \frac{\left(u^{k}\right)_{i+1}^{j}-\left(u^{k}\right)_{i-1}^{j}}{2 h} \\
& \left.+e_{n} \frac{\left(u^{n}\right)_{i+2}^{j}-2\left(u^{n}\right)_{i+1}^{j}+2\left(u^{n}\right)_{i-1}^{j}-\left(u^{n}\right)_{i-2}^{j}}{2 h^{3}}\right)
\end{aligned}
$$


Pick out (in $v$ ) a linear part of expression (B.5) and introduce for convenience an operator $T^{j}$ by the expression

$$
\begin{aligned}
&\left(v^{n}\right)_{i}^{j}-\tau( c_{n} \frac{\left(v^{n}\right)_{i+1}^{j}-\left(v^{n}\right)_{i-1}^{j}}{2 h} \sum_{m, k} g_{m, k}^{n}\left(u^{m}\right)_{i}^{j} \frac{\left(v^{k}\right)_{i+1}^{j}-\left(v^{k}\right)_{i-1}^{j}}{2 h} \\
&+g_{m, k}^{n}\left(v^{m}\right)_{i}^{j} \frac{\left(u^{k}\right)_{i+1}^{j}-\left(u^{k}\right)_{i-1}^{j}}{2 h} \\
&\left.+e_{n} \frac{\left(v^{n}\right)_{i+2}^{j}-2\left(v^{n}\right)_{i+1}^{j}+2\left(v^{n}\right)_{i-1}^{j}-\left(v^{n}\right)_{i-2}^{j}}{2 h^{3}}\right) \\
&=\sum_{r}\left(T^{j+1}\right)_{i r}\left(v^{n}\right)_{i}^{j}, \quad n=1,2,3, \ldots, L .
\end{aligned}
$$

Using the above expression for $T$ and utilizing that $\left(u^{n}\right)_{i}^{j}$ is an exact solution of differential equations, due to the approximation, we use the fact that the right-hand-side term of (B.5) is a small one of order $O\left(\tau+h^{2}\right)$. Therefore, we can rewrite (B.5) as follows:

$$
\begin{aligned}
\left(v^{n}\right)_{i}^{j+1} & -\sum_{r}\left(T^{j+1}\right)_{i r}\left(v^{n}\right)_{i}^{j} \\
& +\tau \sum_{m, k} g_{m, k}^{n}\left(v^{m}\right)_{i}^{j} \frac{\left(v^{k}\right)_{i+1}^{j}-\left(v^{k}\right)_{i-1}^{j}}{2 h}=O\left(\tau+h^{2}\right)
\end{aligned}
$$

or

$$
\left(v^{n}\right)_{i}^{j+1}=\sum_{r}\left(T^{j+1}\right)_{i r}\left(v^{n}\right)_{i}^{j}+\tau\left(f^{n, m, k}\right)_{i}^{j},
$$

where

$$
\left(f^{n m k}\right)_{i}^{j}=-\tau \sum_{m, k} g_{m, k}^{n}\left(v^{m}\right)_{i}^{j} \frac{\left(v^{k}\right)_{i+1}^{j}-\left(v^{k}\right)_{i-1}^{j}}{2 h}+O\left(\tau+h^{2}\right),
$$

and the following estimation for the norm is valid:

$$
\left\|V^{j+1}\right\| \leq\left\|T ^ { j + 1 } \left|\left\||| V^{j}\right\|+\tau\left\|f^{j}\right\|\right.\right.
$$

If we will consequently substitute (B.10) into itself, we will get

$$
\begin{aligned}
& \left\|v^{j+1}\right\| \leq\left\|T^{j+1}||\right\| v^{j}\|+\tau\| f^{j} \| \\
& \leq\left\|T^{j+1}|||| T^{j}|||| v^{j-1}\right\|+\tau\left(\left\|T^{j+1}|||| f^{j-1}\right\|+\left\|f^{j}\right\|\right) \\
& \leq\left\|T^{j+1}|||| T^{j}|||| T^{j-1}|||| v^{j-2}\right\| \\
& +\tau\left(|| T^{j+1}|||| T^{j}|||| f^{j-2}\|+\| T^{j+1}|||| f^{j-1}||+\left\|f^{j}\right\|\right) .
\end{aligned}
$$


Using the estimate of norm of $T$ in inequality (A.5), (B.11) becomes

$$
\begin{aligned}
\left\|v^{j+1}\right\| & \leq e^{a \tau j}\left\|v^{0}\right\|+\tau\left(e^{a \tau(j-1)}\left\|f^{0}\right\|+e^{a \tau(j-2)}\left\|f^{1}\right\|+\cdots\left\|f^{j}\right\|\right) \\
& \leq e^{a \tau j}\left\|v^{0}\right\|+M \max _{k \leq j}\left(\left\|f^{k}\right\|\right), \quad M=\tau \frac{e^{a t_{\max }}-1}{e^{a \tau}-1}
\end{aligned}
$$

$M$ is the sum of a geometric series and $t_{\max }$ is the time of simulation, $0 \leq t \leq$ $t_{\text {max }}$. The norm of $\left(f^{n, m, k}\right)_{i}^{j}$ is given by simple estimations

$$
\begin{aligned}
\left\|f^{j}\right\| & =\left(\sum_{i}\left[\left(f^{m k}\right)_{i}^{j}\right]^{2} h\right)^{1 / 2} \\
& =\max _{n, m, k}\left|g_{m, k}^{n}\right|\left(\sum_{i}\left[\sum_{m, k}\left(v^{m}\right)_{i}^{j} \frac{\left(v^{k}\right)_{i+1}^{j}-\left(v^{k}\right)_{i-1}^{j}}{2 h}\right]^{2} h\right)^{1 / 2} \\
& \leq \max _{n, m, k}\left|g_{m, k}^{n}\right|\left(\sum_{i} \sum_{m}\left[\left(v^{m}\right)_{i}^{j}\right]^{2} h \sum_{i} \sum_{k}\left[\left(v^{k}\right)_{i}^{j}\right]^{2} h\right)^{1 / 2} \frac{1}{h^{3 / 2}} \\
& \leq \frac{\max _{n, m, k}\left|g_{m, k}^{n}\right|}{h^{3 / 2}}\left(\left\|V^{j}\right\|\right)^{2}+O\left(\tau+h^{2}\right) .
\end{aligned}
$$

Using this estimate in inequality (B.12) yields

$$
\left\|V^{j+1}\right\| \leq e^{a \tau j}\left\|V^{0}\right\|+M\left(\frac{\max _{n, m, k}\left|g_{m, k}^{n}\right|}{h^{3 / 2}}\left(\left\|V^{j+1}\right\|\right)^{2}+O\left(\tau+h^{2}\right)\right) .
$$

Taking into consideration $\left\|V^{0}\right\|=0$, the above inequality gives the solution

$$
\begin{aligned}
\left\|V^{j+1}\right\| & \leq \frac{1-\sqrt{1-\left(4 M \max _{n, m, k}\left|g_{m, k}^{n}\right| / h^{3 / 2}\right) M O\left(\tau+h^{2}\right)}}{2 M \max _{n, m, k}\left|g_{m, k}^{n}\right| / h^{3 / 2}} \\
& \cong M O\left(\tau+h^{2}\right),
\end{aligned}
$$

that is, $\left\|V^{j+1}\right\| \rightarrow 0$ as $\tau, h \rightarrow 0$. Hence the convergence is proved.

Acknowledgment. This work was partially supported by the KBN Grant no 5 PO3B 04020.

\section{REFERENCES}

[1] S. P. Kshevetskii, Analytical and numerical investigation of nonlinear internal gravity waves, Nonlinear Processes in Geophysics 8 (2001), 37-53.

[2] P. Lancaster, Theory of Matrices, Academic Press, New York, 1969.

[3] S. B. Leble, Nonlinear Waves in Waveguides: with Stratification, Springer-Verlag, Berlin, 1991.

[4] A. D. McEwan, Internal mixing in stratified fluids, J. Fluid Mech. 128 (1983), 59-80.

[5] _ The kinematics of stratified mixing through internal wavebreaking, J. Fluid Mech. 128 (1983), 47-57. 
[6] Z. Shaohong, A difference scheme for the coupled KdV equation, Commun. Nonlinear Sci. Numer. Simul. 4 (1999), no. 1, 60-63.

[7] J. C. Tannehill, D. A. Anderson, and R. H. Pletcher, Computational Fluid Mechanics and Heat Transfer, Taylor \& Francis, Washington, 1997.

A. A. Halim: Theoretical and Mathematical Physics Department, Gdansk University of Technology, 80-952 Gdansk, Poland

E-mail address: ahmed@mif.pg.gda.p1

S. P. Kshevetskii: Theoretical Physics Department, Kaliningrad State University, Kaliningrad 236041, Russia

E-mail address: verd@tphys.a7bertina.ru

S. B. Leble: Theoretical and Mathematical Physics Department, Gdansk University of Technology, 80-952 Gdansk, Poland

E-mail address: 1eb1e@mif.pg.gda.p1 


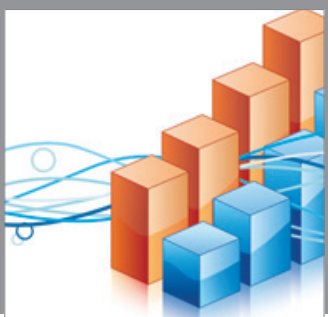

Advances in

Operations Research

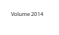

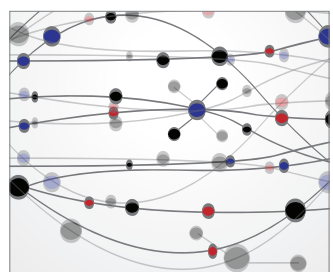

\section{The Scientific} World Journal
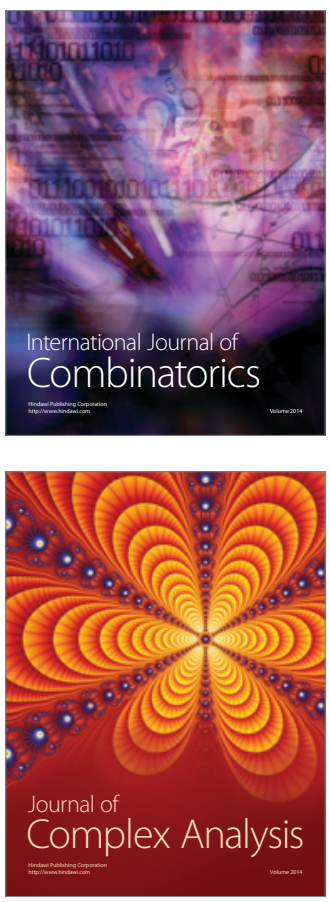

International Journal of

Mathematics and

Mathematical

Sciences
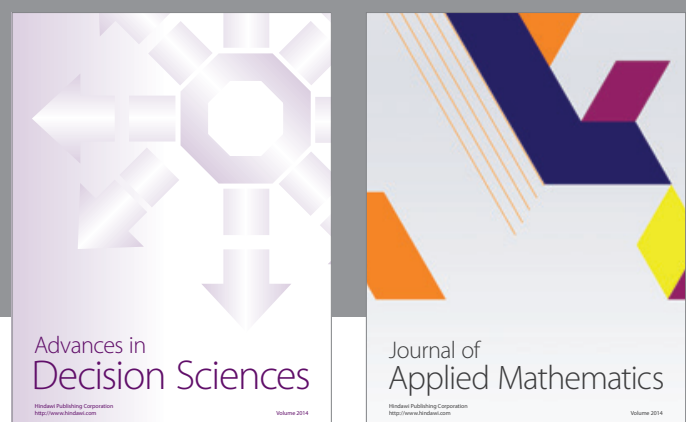

Journal of

Applied Mathematics
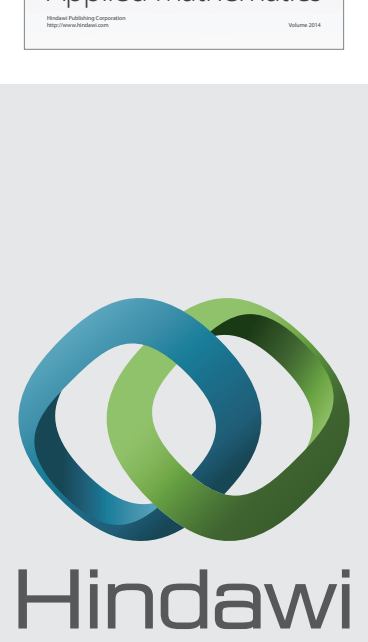

Submit your manuscripts at http://www.hindawi.com
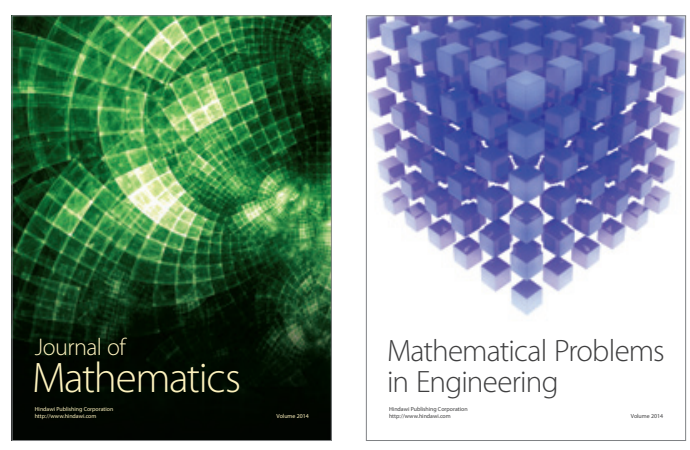

Mathematical Problems in Engineering
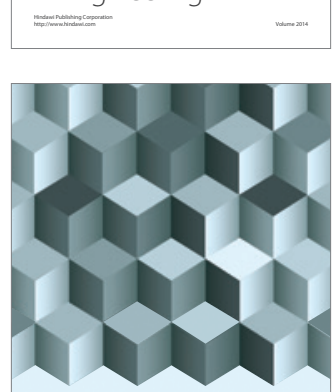

Journal of

Function Spaces
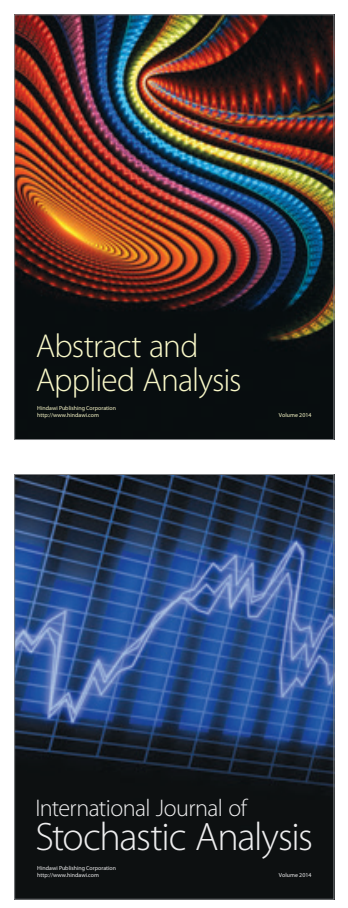

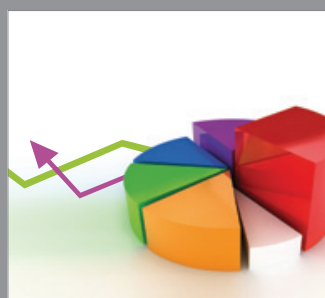

ournal of

Probability and Statistics

Promensencen
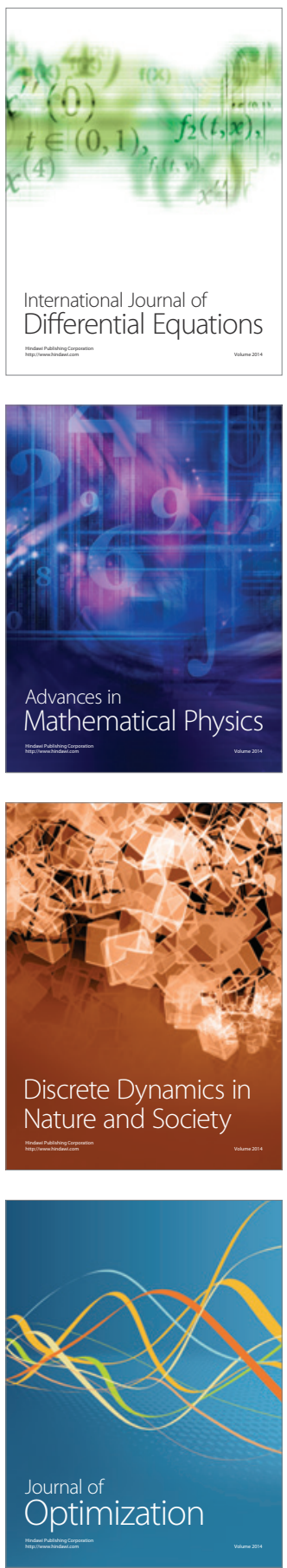